\title{
Application of PSO with Different Typical Neighbor Structure to Complex Job Shop Scheduling Problem
}

\author{
Fuqing Zhao ${ }^{1,2}$, Jianxin Tang ${ }^{1}$, Jizhe Wang ${ }^{1}$, Junbiao Wang ${ }^{2}$ and Jonrinaldi ${ }^{3}$ \\ ${ }^{1}$ School of Computer and Communication, Lanzhou University of Technology, 730050 Gansu,China \\ ${ }^{2}$ Key Laboratory of Contemporary Design \& Integrated Manufacturing Technology,Ministry of Education, Northwestern Polytechnical \\ University,710072 Shanxi, China \\ ${ }^{3}$ School of Engineering, Computer Science and mathematics,University of Exeter,EX4 4QF,United Kingdom
}

\begin{abstract}
Job shop scheduling is to schedule a set of jobs on a set of machines, which is subject to the constraint that each machine can process at most one job at a given time and the fact that each job has a specified processing order through the machines. It is not only a NP-hard problems, it also has the well-earned reputation of being one of the strong combinatorial problems in manufacturing systems. In this paper, the job-shop scheduling problem (JSSP), with the optimization goal of the scheduling problem is minimum of total process time $C_{\max }$, was modeled. An improved particle swarm optimization with acceleration factor (AFPSO) is proposed to improve the ability of particles to explore the global and local optimization solutions, and to reduce the probability of being trapped into the local optima. The neighbor structure of different particle candidate was studied to improve the information exchange speed in optimizing process. Simulation results show that the proposed model and algorithm are effective to task evaluation and implementation.
\end{abstract}

Keywords: Job Shop Scheduling Problem, Particle Swarm Optimization, Topology Structure

\section{Introduction}

Job shop scheduling $[1,2]$ is to schedule a set of jobs on a set of machines, which is subject to the constraint that each machine can process at most one job at a given time and the fact that each job has a specified processing order through the machines. It is not only a NP-hard problems, it also has the well-earned reputation of being one of the strong combinatorial problems in manufacturing systems.

In the previously study, JSSP has been primarily treated by mathematics methods [3], branch and bound methods [4] and heuristics based on priority rules [5]. Over the past two decades, meta-heuristics have gained wide research attention, including such topics as simulated annealing (SA) [6], tabu search [7], genetic algorithm (GA) [8], particle swarm optimization(PSO) [9], and scatter search(SS) [10].

Owing to the good performance of convergence speed in continuous and discrete problems, PSO has been adopted as an optimization method for JSSP for decades. By modifying the particle position representation, particle movement, and particle velocity, Sha [11] constructed a particle swarm optimization (PSO) for an elaborate multi-objective job-shop scheduling problem. A General PSO (GPSO) model, which utilized different typical topology, is adopted by particles to exchange information and search randomly in candidate space. Optimization of the JSP, with a search space division scheme and the meta-heuristic method of PSO, by assigning each machine in a JSP with an independent swarm of particles, was obtained by multiple independent particle swarms [12]. Ivers et al. examines the optimization of the Job Shop Scheduling Problem (JSP) by a search space division scheme and use of the meta-heuristic method of Particle Swarm Optimization (PSO) to solve it [13]. Besides the velocity representation and the guide equations used by the simple PSO, eight extensions focused on the relationship between the particles and the injection of knowledge of the problem was developed to JSP [14]. Many studies have been consequently carried out to prevent premature convergence and to balance the exploration and exploitation abilities [15].

\footnotetext{
*Corresponding author e-mail: fzhao2000@hotmail.com
} 


\section{Job Shop Scheduling Problem}

There are $m$ distinct machines to process $n$ jobs that have their specific processing routines. Each job's operation has its precedence and takes up a deterministic time period at a specific machine. At one time, there is only one operation at a machine and the job does not leave this machine until the operation is completed. Scheduling model is the corresponding evolution process to the initial scheduling model, so the initial problem modeling is as follows.

$$
\min \max \left\{C_{i s} \mid i \in I\right\}
$$

s.t.:

$$
\begin{gathered}
s_{i j+1} \geq s_{i j+1}+p_{i j}, i \in I, J \in\{1, \ldots, s-1\} \\
\left(m_{i_{1} j} \neq m_{i_{2} j}\right) \vee\left(s_{i_{1} j} \geq c_{i_{1} j} \vee s_{i_{2} j} \geq c_{i_{2} j}\right) \\
i_{1}, i_{2} \in I, i_{1} \neq i_{2}, j \in J \\
c_{i j}=s_{i j}+p_{i j}, i \in I, j \in J \\
s_{i_{1}} \geq 0, s_{i j} \geq \sum_{k=1}^{j-1} p_{i k}, i \in I, j \in\{2, \ldots, s\} \\
m_{i j} \in R_{j}=\left\{r_{j l}, \ldots, r_{j l} \mid j \in J\right\}, i \in I
\end{gathered}
$$

Where, $i$ is the workpiece number and $i \in I, I=\{1,2, \cdots, s\}, j$ is the level number and $j \in J, J=\{1,2, \cdots, s\}, r_{j l}$ is the machine number, $s_{i j}$ is the start time of initial scheduling, $m_{i j}$ is the start machine, $p_{i j}$ is the processing time of the workpiece.

\section{The Improved PSO with an Acceleration Factor and Its Performance with Different Topology Structure}

In this paper, an improved PSO algorithm, based on standard PSO algorithm, improves the velocity updating formula by adding an acceleration factor. The modified algorithm can be described as follows:

$$
\left\{\begin{array}{l}
v_{i d}^{t+1}=\omega v_{i d}^{t}+c_{1} r_{1}\left(p_{i d}-x_{i d}^{t}\right)+c_{2} r_{2}\left(p_{g d}-x_{i d}^{t}\right)+l r_{3} \\
x_{i d}^{t+1}=x_{i d}^{t}+v_{i d}^{t+1}
\end{array}\right.
$$

Where, $l=-d_{1}\left(x-d_{2}\right)$, which is a linear decline function controlled by parameters $d_{1}$ and $d_{2}$. The variable $r_{3}$ is a random positive number, drawing from the uniform distribution $[0,1]$. The mathematical model of the dynamic evolutionary equations can be simply described as follows:

$$
\left\{\begin{aligned}
v(t+1)= & \omega v(t)+c_{1} r_{1}\left(p_{i}(t)-x(t)\right)+c_{2} r_{2}\left(p_{g}(t)-x(t)\right) \\
& +l r_{3} \\
x(t+1)= & x(t)+v(t+1)
\end{aligned}\right.
$$

The typical particle topology structure of neighbor domain of particle candidate in PSO has following performance.

Star structure: every particle has direct connection with other members. In this structure, the information exchange rate between particles is very fast, so the convergence speed is fast as well. However, it has the property of the tendency to trap into local optimization in the later evolutions. The topology structure of star structure is shown as Figure 1(a).

Ring structure: all candidates form a ring structure. Each candidate interacts with the neighbor candidate directly to guarantee diversity of the population. The information transfer is slower than that of other structures. The topology structure of ring structure is shown as Figure 1(b).

Rotate structure: each neighbor domain is not formed by 3 candidates. It is formed by a rotate structure with one in center and the rest connected with the one in center. The topology structure of rotate structure is shown as Figure $1(\mathrm{c})$.

Von Neumann structure: each candidate directly connect with the four candidate circled with it to form a net structure. The topology structure of rotate structure is shown as Figure 1(d).

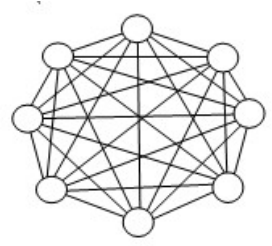

$\rightarrow$ (a) Star structure

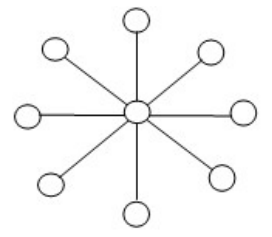

(c) Rotate structure

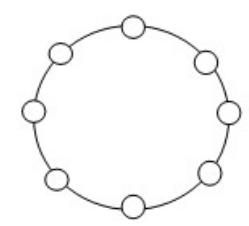

५(b) Ring structure

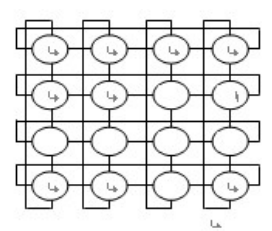

(d) Von Neumann structure
Fig. 1 The neighbor structure of candidate

The following 5 test functions were selected as the test case to the PSO algorithm. In the experiment setup, the 
population size, dimension and iteration number are set 20 , 30 and 1000 , respectively.

$$
\begin{gathered}
f_{1}(X)=\sum_{i=1}^{n}\left[x_{i}^{2}-10 \cos \left(2 \pi x_{i}\right)+10\right],-10<x_{i}<10 \\
f_{2}(X)=\frac{1}{4000} \sum_{i=1}^{n} x_{i}^{2}-\prod_{i=1}^{n} \cos \left(\frac{x_{i}}{\sqrt{i}}\right)+1,-600<x_{i}<600 \\
f_{3}(X)=\sum_{i=1}^{n-1}\left[100\left(x_{i+1}^{2}-x_{i}\right)^{2}+\left(1-x_{i}\right)^{2}\right],-100<x_{i}<100 \\
f_{4}(X)=\sum_{i=1}^{n} x_{i}^{2},-100<x_{i}<100 \\
f_{5}(X)=\sum_{i=1}^{n}\left(\sum_{j=1}^{i} x_{i}^{2}\right),-100<x_{i}<100
\end{gathered}
$$

Table 1 The Optimization Result

$\begin{array}{ccccc}\text { Test } & \text { Topology } & \text { Mean value } & \text { Variance } & \text { Time } \\ 4 * \mathrm{f} 1 & \text { Star } & 42.1271612 & 7.6479676 & 1.469 \\ & \text { Ring } & 31.397248 & 15.0846012 & 0.912 \\ & \text { Rotate } & 50.8840914 & 10.2164443 & 0.796 \\ & \text { Von Neumann } & 69.0705582 & 10.7142657 & 1.914 \\ 4 * \mathrm{f} 2 & \text { Star } & 1.3653234 & 4.4009111 & 0.626 \\ & \text { Ring } & 3.0643189 & 2.429919 & 0.541 \\ & \text { Rotate } & 19.2374623 & 17.2051948 & 0.492 \\ & \text { Von Neumann } & 0.0016375 & 0.0043772 & 0.644 \\ 4 * \mathrm{f} 3 & \text { Star } & 30.2673792 & 21.0242057 & 0.593 \\ & \text { Ring } & 27.3382716 & 0.6353043 & 0.594 \\ & \text { Rotate } & 57.1663611 & 32.6399529 & 0.258 \\ & \text { Von Neumann } & 29.9828141 & 17.1436943 & 0.418 \\ 4 * \mathrm{f} 4 & \text { Star } & 3.469510-17 & 2.324310-16 & 0.68 \\ & \text { Ring } & 0.0000000 & 2.464210-22 & 0.698 \\ & \text { Rotate } & 1.0510-13 & 7.271910-13 & 0.549 \\ & \text { Von Neumann } & 1.27510-15 & 9.015210-15 & 0.64 \\ 4 * f 5 & \text { Star } & 1.397310-15 & 9.800310-15 & 2.089 \\ & \text { Ring } & 0.0000000 & 1.376510-21 & 2.573 \\ & \text { Rotate } & 0.0000000 & 2.535710-27 & 1.856 \\ & \text { Von Neumann } & 4.774410-18 & 3.351310-17 & 2.386\end{array}$

From the Table 1, f1 function has best optimization effect using star topology, but has best stability with star topology. It needs longer running time than that of obtained by other topology because of the neighbor structure. For function $\mathrm{f} 2$, the optimization performance and stability was obtained under von Neumann structure. For function f3, the best optimization was got by star structure. Under star structure, rotate structure and von Neumann structure, f3 can obtain good performance as well. So the neighbor structure has important influence to optimization performance. The optimization result and convergence curves of $\mathrm{f} 1$ and $\mathrm{f} 2$ are shown as Figure 2 (a), (b).

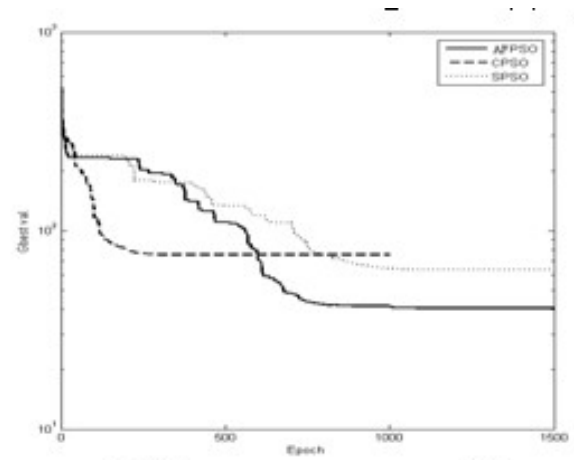

(a) Convergence curves of $\mathrm{fl}$

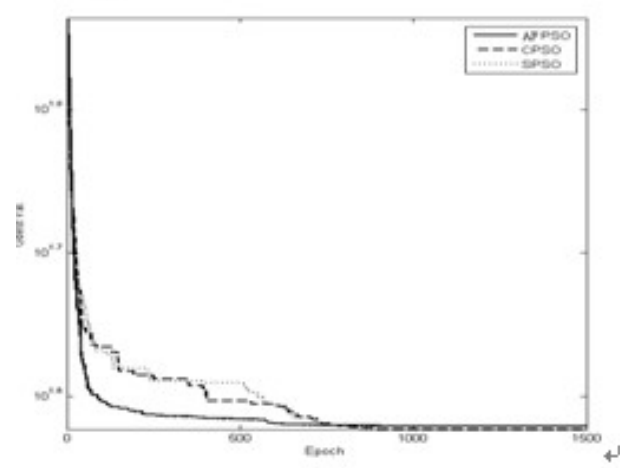

(b) Convergence curves of 12

Fig. 2 Convergence curves

\section{Numerical Test and Comparisons}

To test the performance of the proposed AFPSO, a computational simulation is carried out with some well studied benchmarks. For the problems used as test case, two regular criteria, the makespan $C \max (s)$ and the maximum tardiness $T \max (s)$, are used as criteria. We set the two objectives as follows ( $s$ is the job solution):

Minimize

$$
\begin{aligned}
& f_{1}(s)=C \max (s) \\
& f_{2}(s)=T \max (s)
\end{aligned}
$$

The performances used in the parameters tuning are "Best", "Mean", "Min", and "Best rate", where the "Best", "Mean" and "Min" stand for the best one, the mean one and the minimum aspects of the objective values achieved in 100 runs. The "Best rate" is the rate to reach the best value. The algorithm was run 100 times with different random seeds for each parameter setting to test the random effect on the solution. From the results shown in Table 2, we can see that all the test cases obtained the best value. In order to testify the effective and efficiency of AFPSO, the results reported in Xia and $\mathrm{Wu}$ [16], Sha and Hsu [17] were compared with the results obtained under AFPSO, as shown in Table 2. 
Table 2 Result by using AFPSO

\begin{tabular}{lcccccc}
$2 *$ Test & $2 * \mathrm{nxm}$ & $2 * \mathrm{C} *$ & \multicolumn{4}{c}{ AFPSO } \\
\cline { 4 - 7 } & & & Best & Mean & Min & Time \\
FT06 & $6 \times 6$ & 55 & 55 & 55 & 55 & 6.2 \\
FT10 & $10 \times 10$ & 930 & 930 & 944.5 & 956 & 152.2 \\
FT20 & $20 \times 5$ & 1165 & 1165 & 1182.5 & 1201 & 291.3 \\
LA01 & $10 \times 5$ & 666 & 666 & 666 & 666 & 14.5 \\
LA06 & $15 \times 5$ & 926 & 926 & 926 & 926 & 50.9 \\
LA11 & $20 \times 5$ & 1222 & 1222 & 1222 & 1222 & 129.4 \\
LA16 & $10 \times 10$ & 945 & 945 & 945.5 & 946 & 133.3 \\
LA21 & $15 \times 10$ & 1046 & 1046 & 1052.5 & 1059 & 724.5 \\
LA26 & $20 \times 10$ & 1218 & 1218 & 1218 & 1218 & 2389.6 \\
LA31 & $30 \times 10$ & 1784 & 1784 & 1784 & 1784 & 3698.2 \\
LA36 & $15 \times 15$ & 1268 & 1268 & 1282.4 & 1290 & 3356.2
\end{tabular}

\section{Conclusions}

A modified PSO with an acceleration factor based on basic particle swarm optimization was presented in this paper. The modified algorithm effectively improved the deficiencies of the prone to local optimization solution and slow convergence by adding an acceleration factor to the velocity updating formula of the evolutionary computing algorithm. Theoretical analysis, which is based on stochastic processes, proves that the trajectory of particle is a Markov processes and AFPSO algorithm converges to the global optimal solution with mean square merit. Experimental simulations show that the AFPSO algorithm has better performance on the convergence, and achieves better solutions in shorter time for typical benchmark functions than the standard PSO.

\section{Acknowledgement}

This work is financially supported by the National Natural Science Foundation of China under Grant No.61064011 and 61210306004 . And it was also supported by China Postdoctoral Science Foundation, Science Foundation for The Excellent Youth Scholars of Lanzhou University of Technology, and Educational Commission of Gansu Province of China under Grant No.20100470088, 1014ZCX017 and 1014ZTC090, respectively.

\section{References}

[1] HC Cheng, TC Chiang, LC Fu. A two-stage hybrid memetic algorithm for multiobjective job shop scheduling, Expert Systems with Applications 38. 10983-10998 (2011).

[2] R. Tavakkoli-Moghaddam, M. Azarkish, A. SadeghnejadBarkousaraie. A new hybrid multi-objective Pareto archive PSO algorithm for a bi-objective job shop scheduling problem, Expert Systems with Applications. 10812-10821 (2011).
[3] L. F. Gelders. Sequencing and scheduling - an introduction to the mathematics of the job-shop, European Journal of Operational Research textbf11. 95-96 (1982).

[4] R. Bouabda, B. Jarboui, M. Eddaly, A. Rebai. A branch and bound enhanced genetic algorithm for scheduling a flowline manufacturing cell with sequence dependent family setup times, Computers \& Operations Research 38. 387-393 (2011).

[5] A. Allahverdi, H. Aydilek. Heuristics for the twomachine flowshop scheduling problem to minimise makespan with bounded processing times, International Journal of Production Research 47. 1-19 (2009).

[6] A. Elmi, M. Solimanpur, S. Topaloglu. A simulated annealing algorithm for the job shop cell scheduling problem with intercellular moves and reentrant parts, Computers \& Industrial Engineering 61. 171-178 (2011).

[7] JQ Li, QK Pan, YC Liang. An effective hybrid tabu search algorithm for multi-objective flexible job-shop scheduling problems, Computers \& Industrial Engineering 59. 647-662 (2010).

[8] V. Kachitvichyanukul, S. Sitthitham. A two-stage genetic algorithm for multi-objective job shop scheduling problems, Journal of Intelligent Manufacturing 22. 355-365 (2011).

[9] ZG Lian. A Local and Global Search Combine Particle Swarm Optimization Algorithm for Job-Shop Scheduling to Minimize Makespan, Discrete Dynamics in Nature and Society 2010. 1-11 (2010).

[10] M. M. Nasiri, F. Kianfar. A hybrid scatter search for the partial job shop scheduling problem, International Journal of Advanced Manufacturing Technology 52. 1031-1038 (2011).

[11] D. Y. Sha, L. Hsing-Hung. A multi-objective PSO for jobshop scheduling problems, Expert Systems with Applications 37. 1065-1070 (2010).

[12] B Liu, L Wang, YH Jin. An effective hybrid PSO-based algorithm for flow shop scheduling with limited buffers, Computers \& Operations Research 35. 2791-2806 (2008).

[13] B. Ivers, G. G. Yen. Job Shop Optimization through multiple independent particle swarms, 2007 IEEE Congress on Evolutionary Computation. 3361-3368 (2007).

[14] K. Bulbul. A hybrid shifting bottleneck-tabu search heuristic for the job shop total weighted tardiness problem, Computers \& Operations Research 38. 967-983 (2011).

[15] HW Ge, L Sun, YC Liang, F Qian. An effective PSO and AIS-based hybrid intelligent algorithm for job-shop scheduling, IEEE Transactions on Systems, Man, and Cybernetics-Part A (Systems \& Humans) 38. 358-368 (2008).

[16] WJ Xia, ZM Wu. A hybrid particle swarm optimization approach for the job-shop scheduling problem, International Journal of Advanced Manufacturing Technology 29. 360-366 (2006).

[17] DY Sha, CY Hsu. A hybrid particle swarm optimization for job shop scheduling problem, Computers and Industrial Engineering 51. 791-808 (2006).

Fuqing Zhao, born in 1977, Ph.D of Lanzhou University of Technology. His research interests include theory and application of pattern recognition, computational intelligence and its application. Corresponding author of this paper. 
Jianxin Tang, born in 1985, graduate student of Lanzhou University of Technology. His research interest is the theory and application of pattern recognition and artificial intelligence.

Jizhe Wang, born in 1986, graduate student of Lanzhou University of Technology. His research interest is the theory and application of pattern recognition and artificial intelligence.

Junbiao Wang, born in 1968, professor of Northwestern Polytechnical University. His research interest included integrated manufacturing technology and artificial intelligence.

Jonrinaldi , born in 1977, professor of University of Exeter. His research interest included the swarm intelligence and integrated manufacturing technology. 